Article

\title{
Trapping para-Quinone Methide Intermediates with Ferrocene: Synthesis and Preliminary Biological Evaluation of New Phenol-Ferrocene Conjugates
}

\author{
Silvia González-Pelayo ${ }^{1}$, Enol López ${ }^{1}$, Javier Borge ${ }^{2}$ (D) ${ }^{\text {, Noemí de-los-Santos-Álvarez }}{ }^{3}$ (D) and \\ Luis A. López ${ }^{1, *}$ \\ 1 Departamento de Química Orgánica e Inorgánica and Instituto Universitario de Química Organometálica \\ “Enrique Moles", Universidad de Oviedo, Julián Clavería 8, 33006 Oviedo, Spain; \\ gonzalezpelayos@gmail.com (S.G.-P.); enol.lopezh@gmail.com (E.L.) \\ 2 Laboratorio de Compuestos Organometálicos y Catálisis (Unidad Asociada al CSIC), Centro de Innovación \\ en Química Avanzada (ORFEO-CINQA) and Departamento de Química Física y Analítica, Universidad de \\ Oviedo, Julián Clavería 8, 33006 Oviedo, Spain; jborge@uniovi.es \\ 3 Departamento de Química Física y Analítica, Universidad de Oviedo, Julián Clavería 8, 33006 Oviedo, Spain; \\ santosnoemi@uniovi.es \\ * Correspondence: lalg@uniovi.es; Tel.: +34-985-106-223
}

Received: 9 May 2018; Accepted: 29 May 2018; Published: 1 June 2018

check for updates

\begin{abstract}
The reaction of para-hydroxybenzyl alcohols with ferrocene in the presence of a catalytic amount of $\mathrm{InCl}_{3}$ provided ferrocenyl phenol derivatives, an interesting class of organometallic compounds with potential applications in medicinal chemistry. This transformation exhibited a reasonable substrate scope delivering the desired products in synthetically useful yields. Evidence of involvement of a para-quinone methide intermediate in this coupling process was also provided. Preliminary biological evaluation demonstrated that some of the ferrocene derivatives available by this methodology exhibit significant cytotoxicity against several cancer cell lines with $\mathrm{IC}_{50}$ values within the range of $1.07-4.89 \mu \mathrm{M}$.
\end{abstract}

Keywords: ferrocene; phenol; para-quinone methides; cytotoxic activity

\section{Introduction}

Since the discovery of ferrocene in the 1950s [1,2], the interest in this organometallic compound has not declined. In fact, its chemistry remains one of the most active areas of research. Very likely, this enduring interest resides in the fact that many functionalized ferrocene derivatives display a wide number of applications in a diverse range of fields [3-9]. For example, recent investigations have demonstrated the potential of some ferrocene derivatives in medicinal chemistry $[10,11]$. Particularly, some ferrocene-containing phenols have proved to be of great interest in cancer therapeutics because of their antitumoral activity [12-16]. Among them, a family of ferrocene analogues of hydroxytamoxifen, the so-called ferrocifens (Figure 1a), have been the subject of in-depth investigations showing exceptional cytotoxic activities against some types of breast cancer [17-19]. The mode of action of these organometallic drug candidates has been elucidated by electrochemical and chemical oxidation methods. According to these studies, ferrocenyl quinone methides have been suggested to play a key role in the antiproliferative activity [20-24]. The antitumoral activity of some unconjugated bisphenol derivatives of ferrocene (Figure 1 b) has also been evaluated $[25,26]$. 


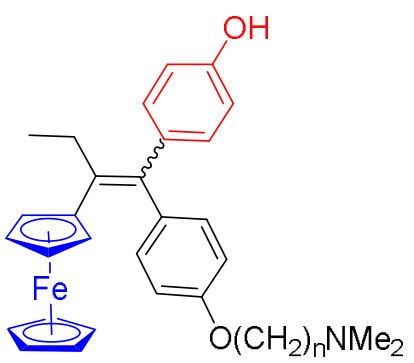

ferrocifens $(n=2-5,8)$ (antitumoral)

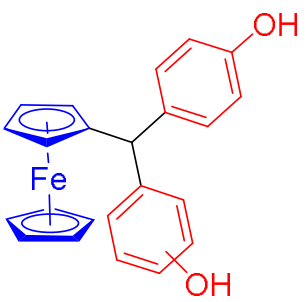

bisphenol ferrocenes

(b)
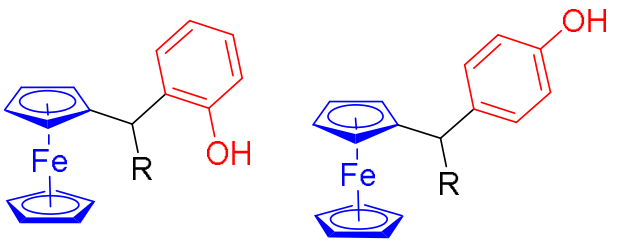

this study (a) (a) Ferrocifen family; (b) ferrocene bisphenol derivatives; (c) ortho-substituted ferrocenyl phenols previously developed in our group; (d) para-substituted ferrocenyl phenols reported in this study.

In connection with our studies on $\mathrm{C}-\mathrm{H}$ bond functionalization of ferrocene based on the trapping of highly electrophilic species [27-29], we have recently described the trapping of ortho-quinone methide intermediates with ferrocene [30]. Interestingly, some of the ferrocene-containing monophenol derivatives available by this methodology (Figure 1c) display remarkable cytotoxic activity against various cancer cell lines.

In order to elucidate the structural requirements for cytotoxicity and, eventually, to identify more bioactive derivatives, we decided to develop a synthetic methodology for the synthesis of the isomeric para-substituted ferrocenylphenol analogues (Figure 1d). Herein, we report the results of this study; specifically, we describe the generation of para-quinone methide intermediates and their trapping with ferrocene. Preliminary biological evaluation of some of the functionalized ferrocene derivatives prepared in this study is also disclosed.

\section{Results and Discussion}

The present study was carried out using easily available $p$-hydroxybenzyl alcohols $1 \mathbf{1}-\mathbf{1}$ outlined in Figure 2.<smiles>[R]C(O)c1ccc(O)cc1</smiles>

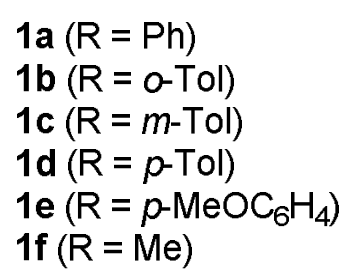

Figure 2. Starting $p$-hydroxybenzyl alcohols 1 used in this work.

For our initial study, $p$-hydroxybenzyl alcohol 1a was chosen as the model substrate (Scheme 1). On the basis of our previous investigations in the ortho series, $\mathrm{InCl}_{3}$ in dichloroethane (DCE) was selected as the catalytic system. Pleasingly, we found that heating a mixture of $\mathbf{1 a}$ (1 equiv.), ferrocene (2, 3 equiv.), and $\mathrm{InCl}_{3}(10 \mathrm{~mol} \%)$ in DCE at $60{ }^{\circ} \mathrm{C}$ led to complete disappearance of the starting $p$-hydroxybenzyl alcohol after $2 \mathrm{~h}$ (checked by thin layer chromatography, TLC). Chromatographic purification $\left(\mathrm{SiO}_{2}, 5: 1\right.$ hexane ethyl acetate) provided the desired functionalized ferrocene derivative $\mathbf{3 a}$ in a remarkable $75 \%$ yield. Interestingly, under otherwise similar conditions, benzydryl alcohol $\mathbf{1} \mathbf{a}^{\prime}$ was found to be a fruitless reaction partner, thus demonstrating the key role of the phenolic $\mathrm{OH}$ group in the reaction course $[31,32]$. 
<smiles>[X]c1ccc(C(O)c2ccccc2-c2ccccc2)cc1</smiles>

$$
1 \mathrm{a}(\mathrm{X}=\mathrm{OH})
$$$$
1 \mathbf{a}^{\prime}(X=H)
$$

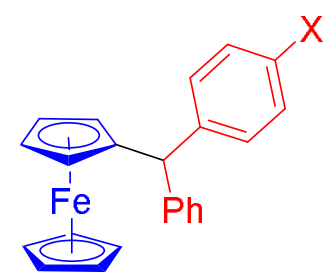

3a $(X=O H, 75 \%)$

$3 a^{\prime}(X=H,<5 \%)$

Scheme 1. Trapping of $p$-quinone methides with ferrocene: proof of concept. DCE: dichloroethane.

Ferrocenyl phenol 3a was characterized by Nuclear Magnetic Resonance (NMR) spectroscopy. Moreover, crystals of compound 3a were obtained from diffusion of pentane into a dichloromethane solution at $-20{ }^{\circ} \mathrm{C}$ and its molecular structure in the solid state has been determined by single-crystal X-ray diffraction (Figure 3 and Appendix A) [33,34]. The electrochemical behavior of compound $3 a$ was investigated by cyclic voltammetry (Appendix B).

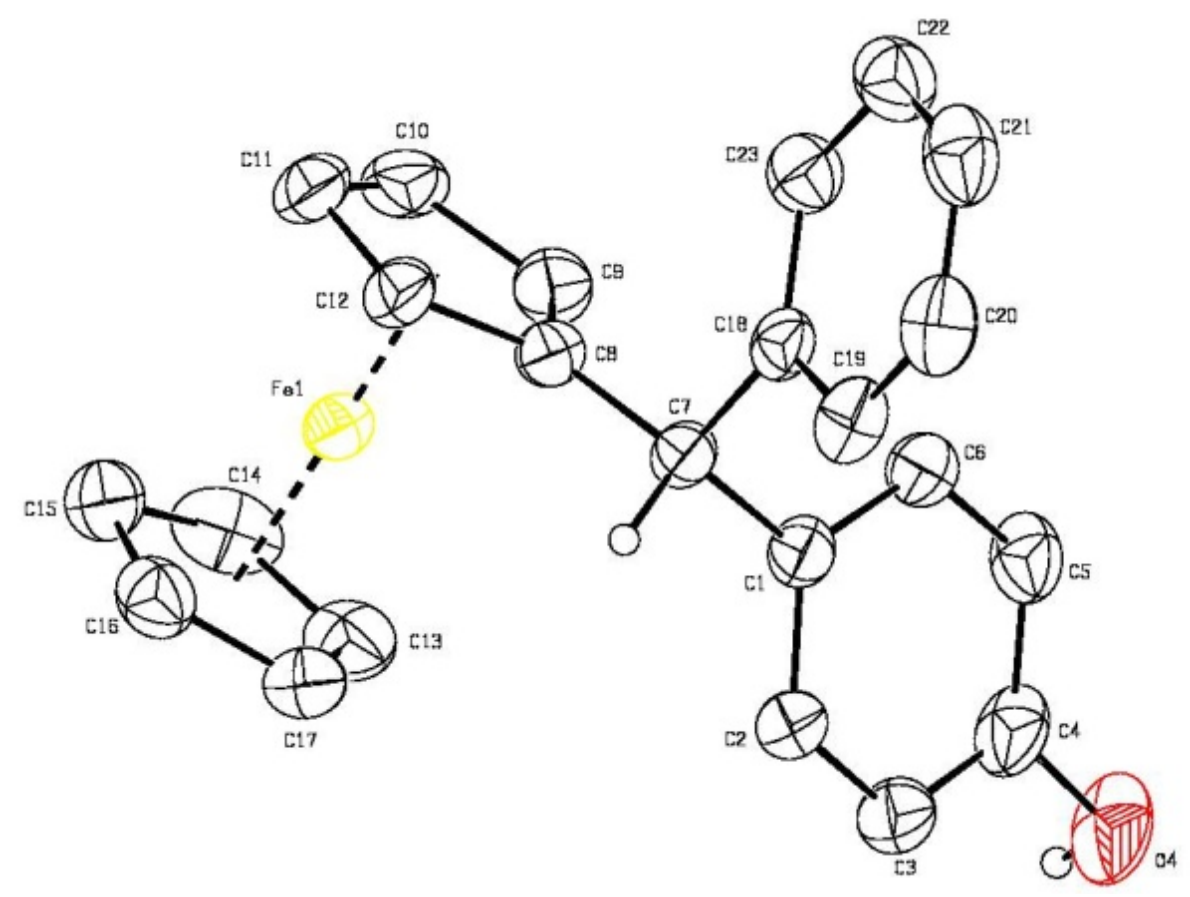

Figure 3. X-ray structure of ferrocene derivative 3a. Thermal ellipsoids are drawn at the $30 \%$ probability level. Hydrogen atoms are excluded, except those bonded to C7 (H7) and $\mathrm{O} 4(\mathrm{H} 4 \mathrm{O})$.

With suitable reaction conditions in hand (10 mol\% of $\mathrm{InCl}_{3}, \mathrm{DCE}$ as solvent, $60{ }^{\circ} \mathrm{C}$ ), a variety of $p$-hydroxybenzyl alcohols were then evaluated for their suitability for this $\mathrm{C}-\mathrm{H}$ bond functionalization process (Table 1). First, some $p$-hydroxybenzyl alcohols substituted with various aryl groups were investigated (entries 2-5). As shown, all three isomeric 4-[hydroxy(tolyl)methyl]phenol derivatives $\mathbf{1 b}-\mathbf{d}(\mathrm{R}=$ tolyl) served as suitable reaction partners for this process furnishing the desired functionalized ferrocene derivatives $3 \mathbf{b}-\mathbf{d}$ in acceptable isolated yields (51-82\%, entries $2-4)$. Similarly, para-methoxy substituted substrate $\mathbf{1 e}\left(\mathrm{R}=p-\mathrm{MeOC}_{6} \mathrm{H}_{4}\right)$ delivered the corresponding product $3 \mathbf{e}$ in moderate isolated yield ( $48 \%$, entry 5$)$. 
Table 1. $\mathrm{InCl}_{3}$-catalyzed reaction of $p$-hydroxybenzyl alcohols $\mathbf{1}$ and ferrocene (2).

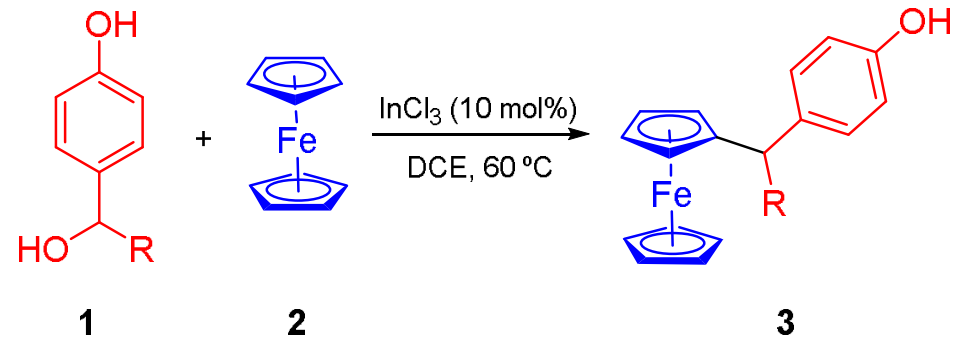

\begin{tabular}{ccccc}
\hline Entry & Substrate & $\mathbf{R}$ & $\mathbf{3}$ & Yield (\%) $^{\mathbf{a}}$ \\
\hline 1 & $\mathbf{1 a}$ & $\mathrm{C}_{6} \mathrm{H}_{5}$ & $\mathbf{3 a}$ & 75 \\
2 & $\mathbf{1 b}$ & $0-\mathrm{MeC}_{6} \mathrm{H}_{4}$ & $\mathbf{3 b}$ & 76 \\
3 & $\mathbf{1 c}$ & $m-\mathrm{MeC}_{6} \mathrm{H}_{4}$ & $3 \mathbf{c}$ & 51 \\
4 & $\mathbf{1 d}$ & $p-\mathrm{MeC}_{6} \mathrm{H}_{4}$ & $\mathbf{3 d}$ & 82 \\
5 & $\mathbf{1 e}$ & $p-\mathrm{MeOC}_{6} \mathrm{H}_{4}$ & $3 \mathbf{e}$ & 48 \\
6 & $\mathbf{1 f}$ & $\mathrm{Me}$ & $\mathbf{3 f}$ & 62 \\
7 & $\mathbf{1 g}$ & $\mathrm{Et}$ & $\mathbf{3 g}$ & 60 \\
8 & $\mathbf{1 h}$ & $n-\mathrm{Bu}$ & $3 \mathbf{h}$ & 42 \\
9 & $\mathbf{1 i}$ & $i-\mathrm{Pr}$ & $3 \mathbf{i}$ & 62 \\
10 & $\mathbf{1 j}$ & $t-\mathrm{Bu}$ & $3 \mathbf{j}$ & 43 \\
11 & $\mathbf{1 k}$ & $\mathrm{Allyl}$ & $3 \mathbf{k}$ & 44 \\
12 & $\mathbf{1 1}$ & $\mathrm{H}$ & $3 \mathbf{l}$ & 64 \\
\hline
\end{tabular}

${ }^{a}$ Isolated yield after chromatographic purification.

Next, substrates $\mathbf{1} \mathbf{f}-\mathbf{j}$ with alkyl groups in the benzylic position were tested (entries 6-10). As shown, primary, secondary and tertiary alkyl groups were well tolerated delivering functionalized ferrocene derivatives $3 \mathbf{f}-\mathbf{j}$ in moderate yields (42-62\%).

This transformation was compatible with unsaturated functional groups in the benzylic position as demonstrated by the synthesis of ferrocene derivative $\mathbf{3 k}$ in moderate yield when substrate $\mathbf{1 k}$ $(\mathrm{R}=$ allyl) was subjected to the standard reaction conditions. Finally, we found that the parent p-hydroxybenzyl alcohol $11(\mathrm{R}=\mathrm{H})$ is also a viable substrate affording the desired product 31 in $64 \%$ yield (entry 12).

Next, to provide further evidence for the involvement of $p$-quinone methide intermediates in the present coupling, we performed an experiment with a stable $p$-quinone methide. Thus, 4-benzylidene-2,6-di-tert-butylcyclohexa-2,5-dienone (4) and ferrocene (2) were subjected to the standard reaction conditions $\left(10 \mathrm{~mol} \%\right.$ of $\left.\mathrm{InCl}_{3}, \mathrm{DCE}, 60{ }^{\circ} \mathrm{C}\right)$. However, a very low conversion was observed after $24 \mathrm{~h}$ very likely due to steric hindrance by the tert-butyl groups. Gratifyingly, performing the reaction in toluene at $100{ }^{\circ} \mathrm{C}$ enabled the preparation of ferrocene derivative 5 in $80 \%$ yield (Scheme 2). Notably, in the absence of $\mathrm{InCl}_{3}$, no reaction occurred at all.<smiles>CC(C)(C)C1=CC(=Cc2ccccc2)C=C(C(C)(C)C)C1=O</smiles>

4

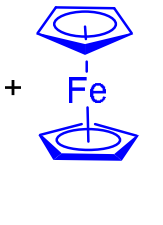

2<smiles>CC(C)(C)c1cc(C(c2ccccc2)c2ccccc2)cc(Br)c1O</smiles>

$5(80 \%)$

Scheme $2 . \mathrm{InCl}_{3}$-catalyzed reaction of ferrocene and stable $p$-quinone methide 4 . 
Based on these control experiments and on previous related literature precedents, a mechanistic proposal for the reaction of hydroxybenzyl alcohols $\mathbf{1}$ and ferrocene (2) is outlined in Scheme 3. In the present process, the Lewis acid is proposed to play a dual role. Firstly, it would promote the generation of the key quinone methide intermediate through dehydration of hydroxybenzyl alcohol 1. Subsequent activation of the quinone methide by Lewis acid complexation would provide intermediate I. This intermediate, with a high electrophilic character at the exocyclic $C=C$ bond, may be involved in a Friedel-Crafts type electrophilic aromatic substitution [35]. Indeed, 1,6-addition of ferrocene would provide the $\sigma$-complex intermediate II, which would evolve to the final product with regeneration of the catalyst [36].

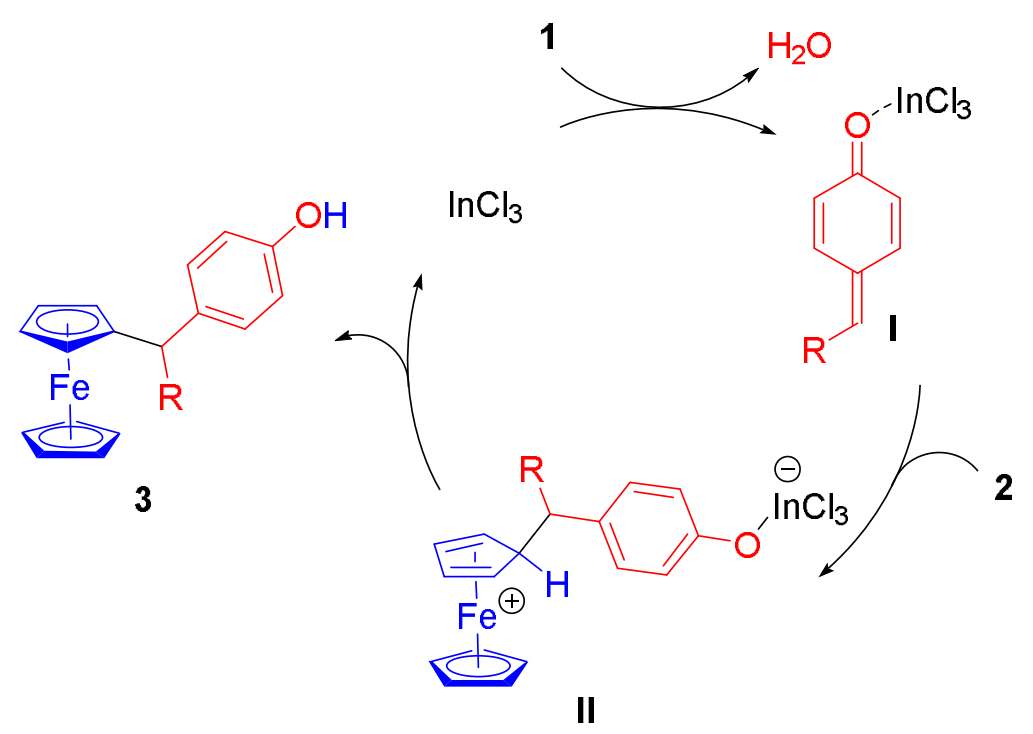

Scheme 3. Mechanism for the $\mathrm{InCl}_{3}$-catalyzed reaction of phenol derivatives $\mathbf{1}$ and ferrocene (2).

Some of the functionalized ferrocene derivatives prepared were evaluated for their cytotoxicity against several cancer cell lines (Table 2). In this preliminary study, ferrocene derivatives $\mathbf{3 a}$ and $\mathbf{3 g}$ were identified as the most active ones [37]. For example, 3a displayed significant toxicity against $\mathrm{A} 2780$ ovarian cancer cell line $\left(\mathrm{IC}_{50}\right.$ of $\left.1.07 \mu \mathrm{M}\right)$. Compared with the value previously reported for the ortho-isomer $\left(\mathrm{IC}_{50}\right.$ of $\left.2.68 \mu \mathrm{M}\right)$, ferrocene derivative $3 \mathrm{a}$ has superior characteristics. Ferrocene $3 \mathrm{~g}$ also displayed toxicity against this cell line $\left(\mathrm{IC}_{50}\right.$ of $\left.2.23 \mu \mathrm{M}\right)$, although somewhat lower than that found for the ortho-analogue $\left(\mathrm{IC}_{50}\right.$ of $\left.1.86 \mu \mathrm{M}\right)$. We have also studied the cytotoxicity profile of ferrocene derivatives 3a and $\mathbf{3 g}$ against A549 lung cancer cells. Both derivatives exhibited moderate cytotoxicity with $\mathrm{IC}_{50}$ values of 3.55 and $4.89 \mu \mathrm{M}$, respectively. These values are comparable to that previously measured for the ortho-isomers ( $\mathrm{IC}_{50}$ of 2.77 and $5.96 \mu \mathrm{M}$, respectively).

Table 2. $\mathrm{IC}_{50}[\mu \mathrm{M}]$ values for selected ferrocenyl compounds on different cell lines ${ }^{\mathrm{a}}$.

\begin{tabular}{ccc}
\hline & 3a & 3g \\
\hline A2780 & 1.07 & 2.23 \\
A549 & 3.55 & 4.89 \\
\hline
\end{tabular}

${ }^{\mathrm{a}}$ Measured after $72 \mathrm{~h}$ of culture. 


\section{Materials and Methods}

\subsection{General}

NMR spectra were recorded at room temperature in $\mathrm{CDCl}_{3}$ on a Bruker DPX-300 or Bruker AVANCE-300 MHz instruments (Bruker, Billerica, MA, USA). Chemical shifts are given in ppm relative to TMS $\left({ }^{1} \mathrm{H}, 0.0 \mathrm{ppm}\right)$ or $\mathrm{CDCl}_{3}\left({ }^{13} \mathrm{C}, 77.0 \mathrm{ppm}\right)$. High-resolution mass spectra were determined on a VG Autospec M mass spectrometer (Waters Corporation, Milford, MA, USA). Cyclic voltammetric studies were performed using a $\mu$-AutoLab type II equipped with GPES 4.9 software (EcoChemie, Utrecht, The Netherlands). All measurements were carried out using a conventional three electrode system in phosphate saline buffer ( $\mathrm{pH}$ 7.4). A modified carbon paste acted as the working electrode and a $\mathrm{Pt}$ wire as a counter electrode. All potentials were referred to a $\mathrm{Ag}|\mathrm{AgCl}| \mathrm{KCl}_{\text {(sat) }}$ reference electrode.

Experiments were carried out under nitrogen using standard Schlenck techniques. 1,2-Dichloroethane was distilled from $\mathrm{CaH}_{2}$. Toluene was distilled from sodium-benzophenone ketyl prior to use. TLC was performed on aluminum-backed plates coated with silica gel 60 with $\mathrm{F}_{254}$ indicator. Flash column chromatography was carried out on silica gel (230-240 mesh). The solvents used in column chromatography, hexane and ethyl acetate, were obtained from commercial suppliers and used without further purification.

p-Hydroxybenzyl alcohols 1a-k were prepared by reaction of 4-hydroxybenzaldehyde with the corresponding Grignard reagents following a literature procedure [38]. p-Hydroxybenzyl alcohol 11 was obtained by the reaction of 4-hydroxybenzaldehyde with $\mathrm{NaBH}_{4}$ [39]. 4-Benzylidene-2,6-di-tert-butylcyclohexa-2,5-dienone (4) was prepared from 2,6-di-tert-butylphenol and benzaldehyde according to a literature procedure [40]. Ferrocene (2) was commercially available and used without further purification.

\subsection{General Procedure for the Synthesis of Ferrocene Derivatives 3a-1}

$\mathrm{InCl}_{3}(4.4 \mathrm{mg}, 0.02 \mathrm{mmol}, 10 \mathrm{~mol} \%$ ) was added to a solution of $p$-hydroxybenzyl alcohols 1 $(0.2 \mathrm{mmol})$ and ferrocene $2(111.6 \mathrm{mg}, 0.6 \mathrm{mmol})$ in 1,2-dichloroethane $(2 \mathrm{~mL})$. The mixture was stirred at $60{ }^{\circ} \mathrm{C}$ for $2-14 \mathrm{~h}$ (disappearance of $\mathbf{1}$ checked by TLC). The solvent was removed under reduced pressure and the resulting residue was purified by flash chromatography (silica gel, mixtures of hexanes/ethyl acetate). Two fractions were collected. The first fraction was unreacted ferrocene and the second one was the corresponding functionalized ferrocene derivative 3. Crystals of compound 3a suitable for X-ray analysis were obtained from diffusion of pentane into dichloromethane at $-20{ }^{\circ} \mathrm{C}$. Copies of ${ }^{1} \mathrm{H}$ - and ${ }^{13} \mathrm{C}$-NMR spectra are provided in the Supplementary Materials.

4-[(Ferrocenyl)(phenyl)methyl]phenol (3a): yellow solid; melting point (m.p.) $63-64{ }^{\circ} \mathrm{C} ;{ }^{1} \mathrm{H}-\mathrm{NMR}$ $\left(300 \mathrm{MHz}, \mathrm{CDCl}_{3}\right): 4.02$ (s, 2H, Cp), 4.05 (s, 5H, Cp), 4.19 (s, 2H, Cp), 4.83 (s, 1H, OH), 5.13 (s, $1 \mathrm{H}, \mathrm{CH}), 6.76(\mathrm{~d}, J=8.1 \mathrm{~Hz}, 2 \mathrm{H}, \mathrm{Ar}), 7.07$ (d, $J=8.1 \mathrm{~Hz}, 2 \mathrm{H}, \mathrm{Ar}), 7.19-7.33$ (m, 5H, Ar); ${ }^{13} \mathrm{C}-\mathrm{NMR}$ (75 MHz, $\left.\mathrm{CDCl}_{3}\right)$ : $51.0(\mathrm{CH}), 67.59(\mathrm{CH}), 67.63(\mathrm{CH}), 68.7(\mathrm{CH}), 92.0(\mathrm{C}), 114.9(\mathrm{CH}), 126.1(\mathrm{CH}), 128.1$ $(\mathrm{CH}), 128.7(\mathrm{CH}), 129.9(\mathrm{CH}), 137.6(\mathrm{C}), 145.3(\mathrm{C}), 153.7(\mathrm{C})$; HRMS (EI) calculated for $\left[\mathrm{C}_{23} \mathrm{H}_{20} \mathrm{FeO}\right]^{+}$ $\left(\mathrm{M}^{+}\right)$: 368.0858, found 368.0856 .

4-[(Ferrocenyl)(2-methylphenyl)methyl]phenol (3b): yellow solid; m.p. $66-67{ }^{\circ} \mathrm{C} ;{ }^{1} \mathrm{H}-\mathrm{NMR}(300 \mathrm{MHz}$, $\left.\mathrm{CDCl}_{3}\right): 2.25$ (s, 3H, CH $), 3.81$ (s, 1H, Cp), 4.07 (s, 5H, Cp), 4.16-4.12 (m, 1H, Cp), 4.20-4.16 (m, 1H, Cp), 4.76 (s, 1H, Cp), 5.25 (s, 1H, CH), 6.78 (d, J = 8.6 Hz, 2H, Ar), 6.96 (s, 1H, OH), 7.09-7.14 (m, 6H, $\mathrm{Ar}) ;{ }^{13} \mathrm{C}-\mathrm{NMR}\left(75 \mathrm{MHz}, \mathrm{CDCl}_{3}\right): 20.3\left(\mathrm{CH}_{3}\right), 47.9(\mathrm{CH}), 67.4(\mathrm{CH}), 68.5(\mathrm{CH}), 68.8(\mathrm{CH}), 69.1(\mathrm{CH}), 69.9$ $(\mathrm{CH}), 93.4(\mathrm{C}), 115.1(\mathrm{CH}), 125.9(\mathrm{CH}), 126.5(\mathrm{CH}), 128.6(\mathrm{CH}), 130.5(\mathrm{CH}), 130.9(\mathrm{CH}), 135.5(\mathrm{C}), 136.0$ (C), $144.7(\mathrm{C}), 154.1(\mathrm{C})$; HRMS (EI) calculated for $\left[\mathrm{C}_{24} \mathrm{H}_{22} \mathrm{FeO}\right]^{+}\left(\mathrm{M}^{+}\right)$: 382.1015, found 382.1009.

4-[(Ferrocenyl)(3-methylphenyl)methyl]phenol (3c): yellow solid; m.p. $72-73{ }^{\circ} \mathrm{C} ;{ }^{1} \mathrm{H}-\mathrm{NMR}(300 \mathrm{MHz}$, $\left.\mathrm{CDCl}_{3}\right): 2.32\left(\mathrm{~s}, 3 \mathrm{H}, \mathrm{CH}_{3}\right), 4.02-4.05(\mathrm{~m}, 7 \mathrm{H}, \mathrm{Cp}), 4.18(\mathrm{~m}, 2 \mathrm{H}, \mathrm{Cp}), 4.62(\mathrm{~s}, 1 \mathrm{H}, \mathrm{OH}), 5.06(\mathrm{~s}, 1 \mathrm{H}, \mathrm{CH})$, $6.75(\mathrm{~d}, J=8.4 \mathrm{~Hz}, 2 \mathrm{H}, \mathrm{Ar}), 7.00-7.17(\mathrm{~m}, 6 \mathrm{H}, \mathrm{Ar}) ;{ }^{13} \mathrm{C}-\mathrm{NMR}\left(75 \mathrm{MHz}, \mathrm{CDCl}_{3}\right): 21.5\left(\mathrm{CH}_{3}\right), 50.9(\mathrm{CH})$, 
$67.7(\mathrm{CH}), 68.9(\mathrm{CH}), 92.3(\mathrm{C}), 114.7(\mathrm{CH}), 120.5(\mathrm{CH}), 125.6(\mathrm{CH}), 126.8(\mathrm{CH}), 127.9(\mathrm{CH}), 129.4(\mathrm{CH})$, $129.9(\mathrm{CH}), 137.5(\mathrm{C}), 145.2(\mathrm{C}), 153.7(\mathrm{C})$; HRMS (EI) calculated for $\left[\mathrm{C}_{24} \mathrm{H}_{22} \mathrm{FeO}\right]^{+}\left(\mathrm{M}^{+}\right)$: 382.1015 , found 382.1011 .

4-[(Ferrocenyl)(4-methylphenyl)methyl]phenol (3d): yellow solid; m.p. 64-65 ${ }^{\circ} \mathrm{C} ;{ }^{1} \mathrm{H}-\mathrm{NMR}(300 \mathrm{MHz}$, $\left.\mathrm{CDCl}_{3}\right): 2.33\left(\mathrm{~s}, 3 \mathrm{H}, \mathrm{CH}_{3}\right), 3.99(\mathrm{~m}, 2 \mathrm{H}, \mathrm{Cp}), 4.03(\mathrm{~s}, 5 \mathrm{H}, \mathrm{Cp}), 4.17(\mathrm{~m}, 2 \mathrm{H}, \mathrm{Cp}), 4.73(\mathrm{~s}, 1 \mathrm{H}, \mathrm{OH}), 5.08(\mathrm{~s}$, $1 \mathrm{H}, \mathrm{CH}), 6.74(\mathrm{~d}, J=8.5 \mathrm{~Hz}, 2 \mathrm{H}, \mathrm{Ar}), 7.04-7.11(\mathrm{~m}, 6 \mathrm{H}, \mathrm{Ar}) ;{ }^{13} \mathrm{C}-\mathrm{NMR}\left(75 \mathrm{MHz}, \mathrm{CDCl}_{3}\right): 21.4\left(\mathrm{CH}_{3}\right)$, $51.0(\mathrm{CH}), 67.9(\mathrm{CH}), 69.1(\mathrm{CH}), 92.6(\mathrm{C}), 115.2(\mathrm{CH}), 128.9(\mathrm{CH}), 129.1(\mathrm{CH}), 130.2(\mathrm{CH}), 135.9(\mathrm{C}), 138.2$ (C), 142.7 (C), 154.1 (C); HRMS (EI) calculated for $\left[\mathrm{C}_{24} \mathrm{H}_{22} \mathrm{FeO}\right]^{+}\left(\mathrm{M}^{+}\right)$: 382.1015, found 382.1009.

4-[(Ferrocenyl)(4-methoxyphenyl)methyl]phenol (3e): yellow oil; ${ }^{1} \mathrm{H}-\mathrm{NMR}\left(300 \mathrm{MHz}, \mathrm{CDCl}_{3}\right): 3.80(\mathrm{~s}, 3 \mathrm{H}$, $\mathrm{OMe}), 3.98$ (s, 2H, Cp), 4.04 (s, 5H, Cp), 4.17 (s, 2H, Cp), 4.72 (s, 1H, CH), 5.06 (s, 1H, OH), 6.79-6.73 (m, 2H, Ar), 6.80-6.84 (m, 2H, Ar), 7.03-7.10 (m, 4H, Ar); ${ }^{13} \mathrm{C}-\mathrm{NMR}\left(75 \mathrm{MHz}, \mathrm{CDCl}_{3}\right): 50.5(\mathrm{CH}), 55.7$ $\left(\mathrm{CH}_{3}\right), 68.0(\mathrm{CH}), 69.2(\mathrm{CH}), 92.9(\mathrm{C}), 113.8(\mathrm{CH}), 115.2(\mathrm{CH}), 130.0(\mathrm{CH}), 130.2(\mathrm{CH}), 138.1(\mathrm{C}), 138.3$ (C), $154.1(\mathrm{C}), 158.2(\mathrm{C})$; HRMS (EI) calculated for $\left[\mathrm{C}_{24} \mathrm{H}_{22} \mathrm{FeO}_{2}\right]^{+}\left(\mathrm{M}^{+}\right)$: 398.0964, found 398.0972.

4-(1-Ferrocenylethyl)phenol (3f): yellow solid; m.p. $=95-96{ }^{\circ} \mathrm{C} ;{ }^{1} \mathrm{H}-\mathrm{NMR}\left(300 \mathrm{MHz}, \mathrm{CDCl}_{3}\right): 1.57(\mathrm{~d}$, $\left.J=7.2 \mathrm{~Hz}, 3 \mathrm{H}, \mathrm{CH}_{3}\right), 3.90(\mathrm{q}, J=7.2 \mathrm{~Hz}, 1 \mathrm{H}, \mathrm{CH}), 4.09$ (s, 1H, Cp), 4.10-4.18 (m, 8H, Cp), $4.63(\mathrm{~s}, 1 \mathrm{H}$, $\mathrm{OH}), 6.74(\mathrm{~d}, J=8.6 \mathrm{~Hz}, 2 \mathrm{H}, \mathrm{Ar}), 7.05(\mathrm{~d}, J=8.6 \mathrm{~Hz}, 2 \mathrm{H}, \mathrm{Ar}) ;{ }^{13} \mathrm{C}-\mathrm{NMR}\left(75 \mathrm{MHz}, \mathrm{CDCl}_{3}\right): 23.1\left(\mathrm{CH}_{3}\right)$, $39.3(\mathrm{CH}), 66.7(\mathrm{CH}), 67.3(\mathrm{CH}), 67.9(\mathrm{CH}), 68.2(\mathrm{CH}), 69.0(\mathrm{CH}), 95.1(\mathrm{C}), 115.4(\mathrm{CH}), 128.6(\mathrm{CH}), 140.4$ (C), 153.9 (C); HRMS (EI) calculated for $\left[\mathrm{C}_{18} \mathrm{H}_{18} \mathrm{FeO}\right]^{+}\left(\mathrm{M}^{+}\right)$: 306.0702 , found 306.0701 . Ferrocene $3 \mathbf{f}$ is a known compound; our characterization data match those previously reported in the literature [25].

4-(1-Ferrocenylpropyl)phenol (3g): yellow solid; m.p. $=87-88^{\circ} \mathrm{C} ;{ }^{1} \mathrm{H}-\mathrm{NMR}\left(300 \mathrm{MHz}, \mathrm{CDCl}_{3}\right): 0.85(\mathrm{t}$, $\left.J=7.4 \mathrm{~Hz}, 3 \mathrm{H}, \mathrm{CH}_{3}\right), 1.67-1.83\left(\mathrm{~m}, 1 \mathrm{H}, \mathrm{CH}_{2}\right), 2.07-2.14\left(\mathrm{~m}, 1 \mathrm{H}, \mathrm{CH}_{2}\right), 3.41-3.46(\mathrm{dd}, J=10.7$ and $4.3 \mathrm{~Hz}$, $1 \mathrm{H}, \mathrm{CH}), 4.06(\mathrm{~s}, 1 \mathrm{H}, \mathrm{Cp}), 4.05-4.10(\mathrm{~m}, 7 \mathrm{H}, \mathrm{Cp}), 4.17(\mathrm{~s}, 1 \mathrm{H}, \mathrm{Cp}), 4.73(\mathrm{~s}, 1 \mathrm{H}, \mathrm{OH}), 6.78(\mathrm{~d}, J=8.5 \mathrm{~Hz}$, 2H, Ar), $7.06(\mathrm{~d}, \mathrm{~J}=8.5 \mathrm{~Hz}, 2 \mathrm{H}, \mathrm{Ar}) ;{ }^{13} \mathrm{C}-\mathrm{NMR}\left(75 \mathrm{MHz}, \mathrm{CDCl}_{3}\right): 12.7\left(\mathrm{CH}_{3}\right), 30.0\left(\mathrm{CH}_{2}\right), 47.0(\mathrm{CH})$, $66.8(\mathrm{CH}), 66.9(\mathrm{CH}), 67.3(\mathrm{CH}), 67.4(\mathrm{CH}), 68.6(\mathrm{CH}), 94.8(\mathrm{C}), 114.9(\mathrm{CH}), 129.0(\mathrm{CH}), 137.8(\mathrm{C}), 153.6$ (C); HRMS (EI) calculated for $\left[\mathrm{C}_{19} \mathrm{H}_{20} \mathrm{FeO}\right]^{+}\left(\mathrm{M}^{+}\right)$: 320.0858 , found 320.0853 .

4-(1-Ferrocenylpentyl)phenol (3h): yellow oil; ${ }^{1} \mathrm{H}-\mathrm{NMR}\left(300 \mathrm{MHz}, \mathrm{CDCl}_{3}\right): 0.89\left(\mathrm{t}, J=7.0 \mathrm{~Hz}, 3 \mathrm{H}, \mathrm{CH}_{3}\right)$, 1.16-1.40 (m, 4H, $\left.\mathrm{CH}_{2}\right), 1.70-1.82\left(\mathrm{~m}, 1 \mathrm{H}, \mathrm{CH}_{2}\right), 2.01-2.08\left(\mathrm{~m}, 1 \mathrm{H}, \mathrm{CH}_{2}\right), 3.53(\mathrm{dd}, J=10.8$ and $4.3 \mathrm{~Hz}$, $1 \mathrm{H}, \mathrm{CH}), 3.95(\mathrm{~s}, 1 \mathrm{H}, \mathrm{Cp}), 4.05-4.11(\mathrm{~m}, 7 \mathrm{H}, \mathrm{Cp}), 4.18(\mathrm{~s}, 1 \mathrm{H}, \mathrm{Cp}), 4.80(\mathrm{~s}, 1 \mathrm{H}, \mathrm{OH}), 6.76(\mathrm{~d}, J=8.3 \mathrm{~Hz}$, 2H, Ar), $7.06(\mathrm{~d}, J=8.3 \mathrm{~Hz}, 2 \mathrm{H}, \mathrm{Ar}) ;{ }^{13} \mathrm{C}-\mathrm{NMR}\left(75 \mathrm{MHz}, \mathrm{CDCl}_{3}\right): 14.1\left(\mathrm{CH}_{3}\right), 22.7\left(\mathrm{CH}_{2}\right), 30.2\left(\mathrm{CH}_{2}\right)$, $36.8\left(\mathrm{CH}_{2}\right), 45.1(\mathrm{CH}), 66.8(\mathrm{CH}), 66.9(\mathrm{CH}), 67.3(\mathrm{CH}), 67.4(\mathrm{CH}), 68.6(\mathrm{CH}), 95.1(\mathrm{C}), 114.9(\mathrm{CH}), 128.9$ $(\mathrm{CH}), 138.1(\mathrm{C}), 153.6(\mathrm{C})$; HRMS (EI) calculated for $\left[\mathrm{C}_{21} \mathrm{H}_{24} \mathrm{FeO}\right]^{+}\left(\mathrm{M}^{+}\right)$: 348.1171, found 348.1184.

4-[1-(Ferrocenyl)(2-methyl)propyl]phenol (3i): yellow solid; m.p. $=95-96{ }^{\circ} \mathrm{C} ;{ }^{1} \mathrm{H}-\mathrm{NMR}\left(300 \mathrm{MHz}, \mathrm{CDCl}_{3}\right)$ : $0.74\left(\mathrm{~d}, J=6.6 \mathrm{~Hz}, 3 \mathrm{H}, \mathrm{CH}_{3}\right), 0.92\left(\mathrm{~d}, J=6.6 \mathrm{~Hz}, 3 \mathrm{H}, \mathrm{CH}_{3}\right), 1.97-2.03(\mathrm{~m}, 1 \mathrm{H}, \mathrm{CH}), 3.08(\mathrm{~d}, J=8.7 \mathrm{~Hz}$, $1 \mathrm{H}, \mathrm{CH}), 3.94(\mathrm{~s}, 5 \mathrm{H}, \mathrm{Cp}), 4.13-4.27(\mathrm{~m}, 4 \mathrm{H}, \mathrm{Cp}), 4.74(\mathrm{~s}, 1 \mathrm{H}, \mathrm{OH}), 6.75(\mathrm{~d}, J=8.3 \mathrm{~Hz}, 2 \mathrm{H}, \mathrm{Ar}), 7.05(\mathrm{~d}$, $J=8.3 \mathrm{~Hz}, 2 \mathrm{H}, \mathrm{Ar}) ;{ }^{13} \mathrm{C}-\mathrm{NMR}\left(75 \mathrm{MHz}, \mathrm{CDCl}_{3}\right): 21.7\left(\mathrm{CH}_{3}\right), 22.2\left(\mathrm{CH}_{3}\right), 53.1(\mathrm{CH}), 66.7(\mathrm{CH}), 67.2$ (CH), $68.4(\mathrm{CH}), 69.3(\mathrm{CH}), 70.4(\mathrm{CH}), 94.7(\mathrm{C}), 114.9(\mathrm{CH}), 129.6(\mathrm{CH}), 137.8(\mathrm{C}), 153.5(\mathrm{C})$; HRMS (EI) calculated for $\left[\mathrm{C}_{20} \mathrm{H}_{22} \mathrm{FeO}\right]^{+}\left(\mathrm{M}^{+}\right)$: 334.1015, found 334.1012.

4-[1-(Ferrocenyl)(2,2-dimethyl)propyl]phenol (3j): yellow solid; m.p. $=105-106{ }^{\circ} \mathrm{C} ;{ }^{1} \mathrm{H}-\mathrm{NMR}(300 \mathrm{MHz}$, $\left.\mathrm{CDCl}_{3}\right): 0.84\left(\mathrm{~s}, 9 \mathrm{H}, \mathrm{CH}_{3}\right), 3.23(\mathrm{~s}, 1 \mathrm{H}, \mathrm{CH}), 3.73(\mathrm{~s}, 5 \mathrm{H}, \mathrm{Cp}), 4.07(\mathrm{~s}, 1 \mathrm{H}, \mathrm{Cp}), 4.14(\mathrm{~d}, J=6.2 \mathrm{~Hz}, 2 \mathrm{H}$, $\mathrm{Cp}), 4.24(\mathrm{~s}, 1 \mathrm{H}, \mathrm{Cp}), 4.93(\mathrm{~s}, 1 \mathrm{H}, \mathrm{OH}), 6.84(\mathrm{~d}, J=8.4 \mathrm{~Hz}, 2 \mathrm{H}, \mathrm{Ar}), 7.27-7.29(\mathrm{~m}, 2 \mathrm{H}, \mathrm{Ar}) ;{ }^{13} \mathrm{C}-\mathrm{NMR}$ $\left(75 \mathrm{MHz}, \mathrm{CDCl}_{3}\right): 28.7\left(\mathrm{CH}_{3}\right), 35.2(\mathrm{C}), 57.3(\mathrm{CH}), 65.7(\mathrm{CH}), 68.0(\mathrm{CH}), 68.1(\mathrm{CH}), 68.5(\mathrm{CH}), 69.2(\mathrm{CH})$, $72.1(\mathrm{CH}), 90.4(\mathrm{C}), 114.2(\mathrm{CH}), 132.0(\mathrm{CH}), 136.9(\mathrm{C}), 153.6(\mathrm{C})$; HRMS (EI) calculated for $\left[\mathrm{C}_{21} \mathrm{H}_{24} \mathrm{FeO}\right]^{+}$ $\left(\mathrm{M}^{+}\right)$: 348.1171, found 348.1182. 
4-[1-(Ferrocenyl)but-3-enyl]phenol (3k): yellow oil; ${ }^{1} \mathrm{H}-\mathrm{NMR}\left(300 \mathrm{MHz}, \mathrm{CDCl}_{3}\right): 2.53-2.62\left(\mathrm{~m}, 1 \mathrm{H}, \mathrm{CH}_{2}\right)$, 2.80-2.89 (m, $\left.1 \mathrm{H}, \mathrm{CH}_{2}\right), 3.65(\mathrm{dd}, J=10.5$ and $4.7 \mathrm{~Hz}, 1 \mathrm{H}, \mathrm{CH}), 4.08(\mathrm{~s}, 1 \mathrm{H}, \mathrm{Cp}), 4.13-4.10(\mathrm{~m}, 7 \mathrm{H}, \mathrm{Cp})$, $4.19(\mathrm{~s}, 1 \mathrm{H}, \mathrm{Cp}), 4.67(\mathrm{~s}, 1 \mathrm{H}, \mathrm{OH}), 4.93-5.04\left(\mathrm{~m}, 2 \mathrm{H},=\mathrm{CH}_{2}\right), 5.76-5.77(\mathrm{~m}, 1 \mathrm{H},=\mathrm{CH}), 6.76(\mathrm{~d}, J=8.6 \mathrm{~Hz}$, $2 \mathrm{H}, \mathrm{Ar}), 7.06(\mathrm{~d}, J=8.6 \mathrm{~Hz}, 2 \mathrm{H}, \mathrm{Ar}) ;{ }^{13} \mathrm{C}-\mathrm{NMR}\left(75 \mathrm{MHz}, \mathrm{CDCl}_{3}\right): 12.7\left(\mathrm{CH}_{3}\right), 30.0\left(\mathrm{CH}_{2}\right), 47.0(\mathrm{CH})$, $66.8(\mathrm{CH}), 66.9(\mathrm{CH}), 67.3(\mathrm{CH}), 67.4(\mathrm{CH}), 68.6(\mathrm{CH}), 94.8(\mathrm{C}), 114.9(\mathrm{CH}), 129.0(\mathrm{CH}), 137.8(\mathrm{C}), 153.6$ (C); HRMS (EI) calculated for $\left[\mathrm{C}_{20} \mathrm{H}_{20} \mathrm{FeO}\right]^{+}\left(\mathrm{M}^{+}\right)$: 332.0858, found 332.0855 .

4-(Ferrocenylmethyl)phenol (31): yellow oil; ${ }^{1} \mathrm{H}-\mathrm{NMR}\left(300 \mathrm{MHz}, \mathrm{CDCl}_{3}\right): 3.64\left(\mathrm{~s}, 2 \mathrm{H}, \mathrm{CH}_{2}\right), 4.11(\mathrm{~s}, 4 \mathrm{H}$, Cp), 4.15 (s, 5H, Cp), $4.84(\mathrm{~s}, 1 \mathrm{H}, \mathrm{OH}), 6.75(\mathrm{~d}, J=8.2 \mathrm{~Hz}, 2 \mathrm{H}, \mathrm{Ar}), 7.02(\mathrm{~d}, J=8.2 \mathrm{~Hz}, 2 \mathrm{H}, \mathrm{Ar}) ;{ }^{13} \mathrm{C}-\mathrm{NMR}$ $\left(75 \mathrm{MHz}, \mathrm{CDCl}_{3}\right)$ : $45.2\left(\mathrm{CH}_{2}\right), 77.6(\mathrm{CH}), 78.7(\mathrm{CH}), 78.8(\mathrm{CH}), 98.7(\mathrm{C}), 125.1(\mathrm{CH}), 139.6(\mathrm{CH}), 144.0$ (C), 163.7 (C); HRMS (EI) calculated for $\left[\mathrm{C}_{17} \mathrm{H}_{16} \mathrm{FeO}\right]^{+}\left(\mathrm{M}^{+}\right)$: 292.0545, found 292.0524. Ferrocene 31 is a known compound [25].

\subsection{Synthesis of Ferrocene Derivative 5}

$\mathrm{InCl}_{3} \quad(4.4 \mathrm{mg}, \quad 0.02 \mathrm{mmol}, \quad 10 \mathrm{~mol} \%)$ was added to a solution of 4-benzylidene-2,6-di-tert-butylcyclohexa-2,5-dienone $4(58.9 \mathrm{mg}, 0.2 \mathrm{mmol})$ and ferrocene 2 $(111.6 \mathrm{mg}, 0.6 \mathrm{mmol})$ in toluene $(2 \mathrm{~mL})$. The mixture was stirred at $100{ }^{\circ} \mathrm{C}$ for $6 \mathrm{~h}$ (disappearance of 4 checked by TLC). Then, the solvent was removed under reduced pressure and the resulting residue was purified by flash chromatography (silica gel, hexanes/ethyl acetate 5:1) to yield ferrocene derivative $5(76.9 \mathrm{mg}, 80 \%)$ as a yellow oil; ${ }^{1} \mathrm{H}-\mathrm{NMR}\left(300 \mathrm{MHz}, \mathrm{CDCl}_{3}\right): 1.44\left(\mathrm{~s}, 18 \mathrm{H}, \mathrm{CH}_{3}\right), 3.98-3.99$ (m, 1H, Cp), 4.01 (s, 5H, Cp), 4.03-4.04 (m, 1H, Cp), 4.16-4.17 (m, 1H, Cp), $4.91(\mathrm{~s}, 1 \mathrm{H}, \mathrm{Cp}), 5.06(\mathrm{~s}, 1 \mathrm{H}$, $\mathrm{CH}), 5.09(\mathrm{~s}, 1 \mathrm{H}, \mathrm{OH}), 7.04(\mathrm{~s}, 2 \mathrm{H}, \mathrm{Ar}), 7.18-7.21(\mathrm{~m}, 3 \mathrm{H}, \mathrm{Ar}), 7.26-7.29(\mathrm{~m}, 2 \mathrm{H}, \mathrm{Ar}){ }^{13} \mathrm{C}-\mathrm{NMR}(75 \mathrm{MHz}$, $\left.\mathrm{CDCl}_{3}\right)$ : $30.0\left(\mathrm{CH}_{3}\right), 34.4(\mathrm{CH}), 51.8(\mathrm{CH}), 67.3(\mathrm{CH}), 67.6(\mathrm{CH}), 68.6(\mathrm{CH}), 68.7(\mathrm{CH}), 68.8(\mathrm{CH}), 92.8$ (C), $125.4(\mathrm{CH}), 125.8(\mathrm{C}), 127.9(\mathrm{CH}), 128.6(\mathrm{CH}), 135.1(\mathrm{C}), 135.4(\mathrm{C}), 143.8(\mathrm{C}), 151.9(\mathrm{C})$; HRMS (EI) calculated for $\left[\mathrm{C}_{31} \mathrm{H}_{36} \mathrm{FeO}\right]^{+}\left(\mathrm{M}^{+}\right)$: 480.2110 , found 480.2124 .

\subsection{Cytotoxic Assays}

Cell Counting Kit-8 (CCK-8) from Sigma-Aldrich (Madrid, Spain) was used according to the protocol provided by the company. The A2780 and A549 cell lines were used in this preliminary study. First, cell lines were cultured for 7 days in Dulbecco's modified Eagle's medium (DMEM) supplemented with $10 \%(v / v)$ fetal bovine serum (FBS). Then, cells were seeded into a 96-well flat-bottom culture plate at a cell density of 500-2000 cells/well and incubated for $24 \mathrm{~h}$ in the same medium (DMEM/10\% FBS). After that, $10 \mu \mathrm{L}$ of a solution of the corresponding ferrocene derivative at different concentrations were added and the cells were incubated for $72 \mathrm{~h}$. Then, $10 \mu \mathrm{L}$ of the CCK-8 solution were added to each well of the plate. After $2 \mathrm{~h}$ of incubation the absorbance at $450 \mathrm{~nm}$ was recorded using a BioTek ELx800 Absorbance Microplate Reader (BioTek, Bad Friedrichshall, Germany). Measurements were performed in triplicate, and each experiment was repeated three times. The $\mathrm{IC}_{50}$ values $(\mu \mathrm{m})$ were estimated by treatment of the data obtained with the statistical program GraphPad Prism5 (version 5.04).

\section{Conclusions}

Guided by earlier work from our group, we have developed a convenient synthesis of para-substituted phenol derivatives containing a ferrocenyl moiety. Salient features of our protocol include (i) easy availability of the required starting materials, (ii) synthetically useful yields, and (iii) mild reaction conditions. This $\mathrm{C}-\mathrm{H}$ bond functionalization of ferrocene relies on the generation of a para-quinone methide intermediate that, activated by Lewis acid complexation, would serve as electrophilic partner in an aromatic electrophilic substitution. Preliminary biological evaluation revealed that some of the ferrocene derivatives available by this protocol display significant cytotoxicity against ovarian and lung cancer cell lines. Further studies aimed at the preparation of new ferrocene derivatives with enhanced antiproliferative properties are being pursued in our laboratory. 
Supplementary Materials: Supplementary Materials available online: Copies of ${ }^{1} \mathrm{H}$ - and ${ }^{13} \mathrm{C}-\mathrm{NMR}$ spectra, X-Ray crystallography (printcif and checkcif).

Author Contributions: L.A.L. conceived the experiments; S.G.-P. and E.L. designed and performed the experiments; J.B. performed the X-ray crystallographic study; N.d.-1.-S.-A. performed the voltammetry study; L.A.L., J.B. and N.d.-1.-S.-A. wrote the paper.

Acknowledgments: Financial support from the Ministerio de Economía y Competitividad (MINECO, grants CTQ2013-41511-P and CTQ2015-63567-R), Agencia Estatal de Investigación (AEI) and Fondo Europeo de Desarrollo Regional (FEDER) (Grants CTQ2016-76840-R and CTQ2016-75986-P), and Principado de Asturias (grants GRUPIN14-013 and GRUPIN14-006) is gratefully acknowledged. E.L. thanks the Principado de Asturias for a predoctoral grant (Severo Ochoa Program). We also thank J.M. González for interesting discussions.

Conflicts of Interest: The authors declare no conflict of interest.

\section{Appendix A. Crystal Data for Ferrocene Derivative 3a}

Crystal Data for $\mathrm{C}_{23} \mathrm{H}_{20} \mathrm{FeO}\left(M_{\mathrm{r}}=368.24 \mathrm{~g} / \mathrm{mol}\right)$ : monoclinic, space group $P 2_{1} / \mathrm{n}$ (No. 14), $a=$ 15.662(1) $\AA, b=6.0219(3) \AA, c=18.786(1) \AA, \beta=96.990(6)^{\circ}, V=1758.6(2) \AA^{3}, Z=4, T=299 \mathrm{~K}, \mu(\mathrm{CuK \alpha})=$ $6.91 \mathrm{~mm}^{-1}, \mathrm{D}_{\mathrm{x}}=1.391 \mathrm{~g} / \mathrm{cm}^{3}, 8336$ measured reflections $\left(3.5^{\circ}<\theta<69.6^{\circ}\right), 3254$ independent reflections, 2641 observed reflections $(I>2 \sigma(I)), R_{\text {int }}=0.045$. Final $R\left[F^{2}>2 \sigma\left(F^{2}\right)\right]$ was 0.079 and $w R\left(F^{2}\right)=0.255$.

\section{Appendix B. Electrochemical Study for Ferrocene Derivative 3a}

Compound 3a was studied by cyclic voltammetry $(\mathrm{CV})$. All potentials were referred to a $\mathrm{Ag}|\mathrm{AgCl}| \mathrm{KCl}_{\text {(sat) }}$ reference electrode. The ferrocene/ferrocenium couple $\left(\mathrm{I}_{\mathrm{a}} / \mathrm{I}_{\mathrm{c}}\right)$ is clearly observed at a formal potential, $\mathrm{E}^{\circ \prime}=0.352 \mathrm{~V}$ (Figure A1a). The anodic peak current is higher than the cathodic one indicating that some ferrocenium ions diffuse from the carbon paste electrode to the bulk solution due to the positive charge of the cation. Nucleation at potentials $\sim 0.450 \mathrm{~V}$ points out to a dissimilar electrochemical behavior with other structurally related compounds. When the potential is swept up to $+0.6 \mathrm{~V}$, a second redox process $\left(\mathrm{II}_{\mathrm{a}} / \mathrm{II}_{\mathrm{c}}\right.$ ) appears causing the decrease of $\mathrm{I}_{\mathrm{a}}$ (Figure $\mathrm{A} 1 \mathrm{~b}$ ). The reduction of ferrocenium species $\left(\mathrm{I}_{\mathrm{C}}\right)$ remains visible but progressively shifted towards less positive potentials in subsequent scans. Process $\mathrm{II}_{\mathrm{a}}$ at about $+0.570 \mathrm{~V}$ is clear and well-shaped but process $\mathrm{II}_{\mathrm{c}}$ at $0.426 \mathrm{~V}$ is partially overlapped with $\mathrm{I}_{\mathrm{c}}$. Of note, a notably increase in the non-faradaic current is observed. When the potential is extended to $+1.3 \mathrm{~V}$, additional oxidation reactions take place (see the rising anodic current) probably associated to phenolic compounds. Only one reduction process is observed as a result of $\mathrm{I}_{\mathrm{c}}$ and $\mathrm{II}_{\mathrm{c}}$ overlapping (Figure A1c). The potential of both oxidation and reduction peaks shift to more extreme potentials which indicates that the process is irreversible. The origin of the irreversibility might be the formation of non-conducting products on the electrode surface that hinder the electron transfer. The appearance of resistance affecting the CV shape strongly supports this explanation. 

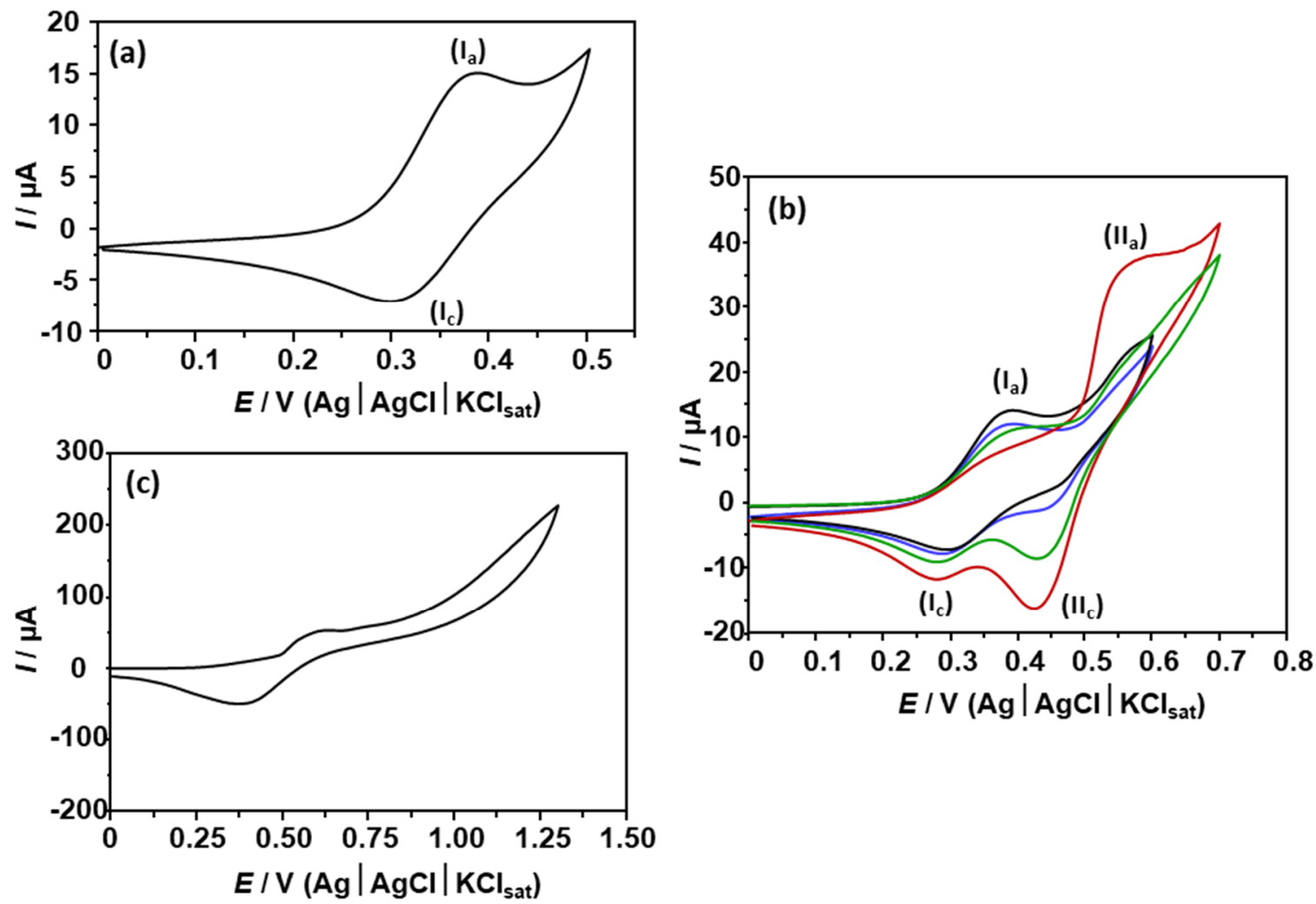

Figure A1. Cyclic voltammetry of compound 3a incorporated to the carbon paste electrode in phosphate saline buffer ( $\mathrm{pH}$ 7.4). Scan rate $=50 \mathrm{mV} / \mathrm{s}$. Potential scan from $0 \mathrm{~V}$ to $+0.5 \mathrm{~V}$; (a) +0.6 (first scan, black; second scan, blue) and +0.7 (first scan, green; second scan, red) (b) and $+1.3 \mathrm{~V}$ (c).

\section{References and Notes}

1. Kealy, T.J.; Pauson, P.L. A New Type of Organo-Iron Compound. Nature 1951, 168, 1039-1040. [CrossRef]

2. Miller, S.A.; Tebboth, J.A.; Tremaine, J.F. Dicyclopentadienyliron. J. Chem. Soc. 1952, 632-635. [CrossRef]

3. Štěpnička, P. Ferrocenes: Ligands, Materials and Biomolecules; Wiley: Chichester, UK, 2008.

4. Phillips, E.S. Ferrocenes: Compounds, Properties and Applications; Nova Science Publishers: New York, NY, USA, 2011.

5. Ferrocenes. Homogeneous Catalysis. Organic Synthesis. Materials Science; Togni, A., Hayashi, T., Eds.; VCH: Weinheim, Germany, 1995.

6. Chiral Ferrocenes in Asymmetric Catalysis; Dai, L.-X., Hou, X.-L., Eds.; Wiley-VCH: Weinheim, Germany, 2009.

7. Astruc, D. Why is Ferrocene so Exceptional? Eur. J. Inorg. Chem. 2017, 1, 6-29. [CrossRef]

8. Arrayás, R.G.; Adrio, J.; Carretero, J.C. Recent Applications of Chiral Ferrocene Ligands in Asymmetric Catalysis. Angew. Chem. Int. Ed. 2006, 45, 7674-7715. [CrossRef] [PubMed]

9. Van Staveren, D.R.; Metzler-Nolte, N. Bioorganometallic Chemistry of Ferrocene. Chem. Rev. 2004, 104, 5931-5986. [CrossRef] [PubMed]

10. Patra, M.; Gasser, G. The medicinal chemistry of ferrocene and its derivatives. Nat. Rev. Chem. 2017, 1, 066. [CrossRef]

11. Dive, D.; Biot, C. Ferroquine as an Oxidative Shock Antimalarial. Curr. Top. Med. Chem. 2014, 14, $1684-1692$. [CrossRef] [PubMed]

12. Braga, S.S.; Silva, A.M.S. A New Age for Iron: Antitumoral Ferrocenes. Organometallics 2013, 32, 5626-5639. [CrossRef]

13. Melendez, E. Metallocenes as target specific drugs for cancer treatment. Inorg. Chim. Acta 2012, 393, 36-52. [CrossRef] [PubMed]

14. Ornelas, C. Application of ferrocene and its derivatives in cancer research. New J. Chem. 2011, 35, 1973-1985. [CrossRef] 
15. Schatzschneider, U.; Metzler-Nolte, N. New Principles in Medicinal Organometallic Chemistry. Angew. Chem. Int. Ed. 2006, 45, 1504-1507. [CrossRef] [PubMed]

16. Gasser, G.; Ott, I.; Metzler-Nolte, N. Organometallic Anticancer Compounds. J. Med. Chem. 2011, 54, 3-25. [CrossRef] [PubMed]

17. Jaouen, G.; Vessières, A.; Top, S. Ferrocifen type anti cancer drugs. Chem. Soc. Rev. 2015, 44, 8802-8817. [CrossRef] [PubMed]

18. Nguyen, A.; Vessières, A.; Hillard, E.A.; Top, S.; Pigeon, P.; Jaouen, G. Ferrocifens and ferrocifenols as new potential weapons against breast cancer. Chimia 2007, 61, 716-724. [CrossRef]

19. Vessières, A. Metal carbonyl tracers and the ferrocifen family: Two facets of biorganometallic chemistry. J. Organomet. Chem. 2013, 734, 3-16. [CrossRef]

20. Hillard, E.A.; Vessières, A.; Jaouen, G. Ferrocene functionalized endocrine modulators as anticancer agents. Top. Organomet. Chem. 2010, 32, 81-117.

21. Hillard, E.; Vessières, A.; Thouin, L.; Jaouen, G.; Amatore, C. Ferrocene-mediated proton-coupled electron transfer in a series of ferrocifen-type breast-cancer drug candidates. Angew. Chem. Int. Ed. 2006, 45, 285-290. [CrossRef] [PubMed]

22. Hamels, D.; Dansette, P.M.; Hillard, E.A.; Top, S.; Vessières, A.; Herson, P.; Jaouen, G.; Mansuy, D. Ferrocenyl quinone methides as strong antiproliferative agents: Formation by metabolic and chemical oxidation of ferrocenyl phenols. Angew. Chem. Int. Ed. 2009, 48, 9124-9126. [CrossRef] [PubMed]

23. Messina, P.; Labbé, E.; Buriez, O.; Hillard, E.A.; Vessières, A.; Hamels, D.; Top, S.; Jaouen, G.; Frapart, Y.M.; Mansuy, D.; et al. Deciphering the activation sequence of ferrociphenol anticancer drug candidates. Chem. Eur. J. 2012, 18, 6581-6587. [CrossRef] [PubMed]

24. Wang, Y.; Pigeon, P.; Top, S.; McGlinchey, M.J.; Jaouen, G. Organometallic antitumor compounds: ferrocifens as precursors to quinone methides. Angew. Chem. Int. Ed. 2015, 54, 10230-10233. [CrossRef] [PubMed]

25. Hillard, E.; Vessières, A.; Le Bideau, F.; Plazuk, D.; Spera, D.; Huché, M.; Jaouen, G. A Series of Unconjugated Ferrocenyl Phenols: Prospects as Anticancer Agents. Chem. Med. Chem. 2006, 1, 551-559. [CrossRef] [PubMed]

26. Plazuk, D.; Vessières, A.; Le Bideau, F.; Jaouen, G.; Zakrzewski, J. Synthesis of Benzyl- and Benzhydrylferrocenes via Friedel-Crafts Alkylation of Ferrocene. Access to Ferrocenyl Bisphenols with High Affinities for Estrogen Receptors. Tetrahedron Lett. 2004, 45, 5425-5427. [CrossRef]

27. López, E.; Lonzi, G.; López, L.A. Gold-Catalyzed C-H Bond Functionalization of Metallocenes: Synthesis of Densely Functionalized Ferrocene Derivatives. Organometallics 2014, 33, 5924-5927. [CrossRef]

28. López, E.; Borge, J.; López, L.A. Gold-Catalyzed Intermolecular Formal Insertion of Aryldiazo Esters into Cp-H Bonds of Iron and Ruthenium Metallocenes. Chem. Eur. J. 2017, 23, 3091-3097. [CrossRef] [PubMed]

29. López, E.; Suárez, T.; Ballesteros, A.; López, L.A. Gold(I)-Catalyzed Reaction of Ferrocene and Propargylic Esters: Synthesis of Functionalized Ferrocene Derivatives. Eur. J. Inorg. Chem. 2017, 225-228. [CrossRef]

30. González-Pelayo, S.; López, E.; Borge, J.; de-los-Santos-Álvarez, N.; López, L.A. Ferrocene-Decorated Phenol Derivatives by Trapping of ortho-Quinone Methide Intermediates with Ferrocene. Eur. J. Org. Chem. 2018. [CrossRef]

31. The Lewis acid-catalyzed monoalkylation of ferrocene with some benzyl alcohols has been reported. This process takes place in molten ferrocene as solvent at high temperature $\left(176{ }^{\circ} \mathrm{C}\right)$ : Abdullah, R.; Chung, $\mathrm{K}$.; Kim, Y.-W.; Hong, I.S. An Efficient Synthesis of Mono-Substituted Benzyl Ferrocene Derivatives. Bull. Korean Chem. Soc. 2015, 36, 32-35. [CrossRef]

32. For a similar methodology for the alkylation of ferrocene, see: Neuse, E.W.; Trifan, D.S. Alkylation of Ferrocene with $\alpha$-Aryl Alcohols. J. Am. Chem. Soc. 1962, 84, 1850-1856.

33. COD 3000199 Contains the Supplementary Crystallographic Data for This Paper. These Data Can Be Obtained Free of Charge via. See also Supplementary Materials. Available online: http://www. crystallography.net/search.html (accessed on 30 May 2018).

34. A detailed analysis revealed a non-classical intermolecular interaction of the phenolic OH group with the $\pi$ cloud of the aromatic ring. For selected examples of aromatic rings as weak hydrogen bond acceptors in crystals, see: Jeffrey, G.A. An Introduction to Hydrogen Bonding; Oxford University Press: New York, NY, USA, 1997.

35. Seminal contribution on Friedel-Crafts acylation of ferrocene: Woodward, R.B.; Rosenblum, M.; Whiting, M.C. A new aromatic system. J. Am. Chem. Soc. 1952, 74, 3458-3459. [CrossRef] 
36. For a recent related arylation process involving electron-rich arenes, see: Gao, S.; Xu, X.; Yuan, Z.; Zhou, H.; Yao, H.; Lin, A. 1,6-Addition arylation of para-quinone methides: An approach to unsymmetrical triarylmethanes. Eur. J. Org. Chem. 2016, 2016, 3006-3012. [CrossRef]

37. In this study, NIH $3 \mathrm{~T} 3$ cells were used as normal control cell line.

38. Wong, Y.F.; Wang, Z.; Sun, J. Chiral phosphoric acid catalyzed asymmetric addition of naphthols to para-quinone methides. Org. Biomol. Chem. 2016, 14, 5751-5754. [CrossRef] [PubMed]

39. McNulty, J.; McLeod, D. A scalable process for the synthesis of (E)-Pterostilbene involving aqueous Wittig olefination chemistry. Tetrahedron Lett. 2013, 54, 6303-6306. [CrossRef]

40. Chu, W.-D.; Zhang, L.-F.; Bao, X.; Zhao, X.-H.; Zeng, C.; Du, J.-Y.; Zhang, G.-B.; Wang, F.-X.; Ma, X.-Y.; Fan, C.-A. Asymmetric catalytic 1,6-conjugate addition/aromatization of para-quinone methides: Enantioselective introduction of functionalized diarylmethine sterogenic centers. Angew. Chem. Int. Ed. 2013, 52, 9229-9233. [CrossRef] [PubMed]

Sample Availability: Samples of the compounds are available from the authors.

(C) 2018 by the authors. Licensee MDPI, Basel, Switzerland. This article is an open access article distributed under the terms and conditions of the Creative Commons Attribution (CC BY) license (http://creativecommons.org/licenses/by/4.0/). 